\title{
Recognizing Syntactic Errors in the Writing of Second Language Learners*
}

\author{
David Schneider \\ Department of Linguistics \\ University of Delaware \\ Newark, DE 19716 \\ and \\ Kathleen F. McCoy \\ Computer and Information Sciences \\ University of Delaware \\ Newark, DE 19716 \\ \{dschneid,mccoy\}@cis.udel.edu
}

\begin{abstract}
This paper reports on the recognition component of an intelligent tutoring system that is designed to help foreign language speakers learn standard English. The system models the grammar of the learner, with this instantiation of the system tailored to signers of American Sign Language (ASL). We discuss the theoretical motivations for the system, various difficulties that have been encountered in the implementation, as well as the methods we have used to overcome these problems. Our method of capturing ungrammaticalities involves using malrules (also called 'error productions'). However, the straightforward addition of some mal-rules causes significant performance problems with the parser. For instance, the ASL population has a strong tendency to drop pronouns and the auxiliary verb 'to be'. Being able to account for these as sentences results in an explosion in the number of possible parses for each sentence. This explosion, left unchecked, greatly hampers the performance of the system. We discuss how this is handled by taking into account expectations from the specific population (some of which are captured in our unique user model). The different representations of lexical items at various points in the acquisition process are modeled by using mal-rules, which obviates the need for multiple lexicons. The grammar is evaluated on its ability to correctly diagnose agreement problems in actual sentences produced by ASL native speakers.
\end{abstract}

\section{Overview}

This paper reports on the error-recognition component of the ICICLE (Interactive Computer Identification and Correction of Language Errors) system. The system is designed to be a tutorial system for helping second-language (L2) learners of English. In this instantiation

\footnotetext{
* This work was supported by NSF Grant \#SRS9416916.
}

of the system, we are focusing on the particular problems of American Sign Language (ASL) native signers. The system recognizes errors by using mal-rules (also called 'errorproduction rules') (Sleeman, 1982), (Weischedel et al., 1978) which extend the language accepted by the grammar to include sentences containing the specified errors. The mal-rules themselves are derived from an error taxonomy which was the result of an analysis of writing samples. This paper focuses primarily on the unique challenges posed by developing a grammar that allows the parser to efficiently parse and recognize errors in sentences even when multiple errors occur. Additionally, it is important to note that the users will not be at a uniform stage of acquisition - the system must be capable of processing the input of users with varying levels of English competence. We briefly describe how acquisition is modeled and how this model can help with some of the problems faced by a system designed to recognize errors.

We will begin with an overview of the entire ICICLE system. To motivate some of the difficulties encountered by our mal-rule-based error recognition system, we will briefly describe some of the errors common to the population under study. A major problem that must be faced is parsing efficiency caused by multiple parses. This is a particularly difficult problem when expected errors include omission errors, and thus this class of errors will be discussed in some detail. Another important problem involves the addition/subtraction of various syntactic features in the grammar and lexicon during acquisition. We describe how our system models this without the use of multiple lexicons. We follow this by a description of the current implementation and grammar coverage of the system. Finally, we will present an evaluation of the system for number/agreement errors in the target group of language learners. 


\section{System Overview}

The ICICLE system is meant to help secondlanguage learners by identifying errors and engaging the learners in a tutorial dialogue. It takes as input a text written by the student. This is given to the error identification component, which is responsible for flagging the errors. The identification is done by parsing the input one sentence at a time using a bottomup chart parser which is a successor to (Allen, 1995). The grammar formalism used by the parser consists of context-free rules augmented with features. The grammar itself is a grammar of English which has been augmented with a set of mal-rules which capture errors common to this user population. We will briefly discuss some classes of errors that were uncovered in our writing sample analysis which was used to identify errors expected in this population. This discussion will motivate some of the mal-rules which were written to capture some classes of errors, and the difficulties encountered in implementing these mal-rules. The mal-rules are specially tagged with information helpful in the correction phase of the system.

The error identification component relies on information in the user model - the most interesting aspect of which is a model of the acquisition of a second language. This model (instantiated with information from the ASL/English language model) is used to highlight those grammar rules which the student has most likely already acquired or is currently in the process of acquiring. These rules will be the ones the parser attempts to use when parsing the user's input. Thus we take an interlanguage view of the acquisition process (Selinker, 1972), (Ellis, 1994), (Cook, 1993) and attempt to model how the student's grammar is likely to change over time. The essence of the acquisition model is that there are discrete stages that all learners of a particular language will go through (Krashen, 1981), (Ingram, 1989), (Dulay and Burt, 1974), (Bailey et al., 1974). Each of these stages is characterized in our model by sets of language features (and therefore constructions) that the learner is in the process of acquiring. It is anticipated that most of the errors that learners make will be within the constructions (where "construction" is construed broadly) that they are in the process of acquiring (Vygotsky, 1986) and that they will favor sentences involving those constructions in a "hypothesize and test" style of learning, as predicted by interlanguage theory. Thus, the parser favors grammar rules involving constructions currently being acquired (and, to a lesser extent, constructions already acquired).

The correction phase of the system is a focus of current research. A description of the strategies for this phase can be found in (Michaud and McCoy, 1998) and (Michaud, 1998).

\section{Expected Errors}

In order to identify the errors we expect the population to make, we collected writing samples from a number of different schools and organizations for the deaf. To help identify any instances of language transfer between ASL and written English, we concentrated on eliciting samples from deaf people who are native ASL signers. It is important to note that ASL is not simply a translation of standard English into manual gestures, but rather is a complete language with its own syntax, which is significantly different from English. Some of our previous work (Suri and McCoy, 1993) explored how language transfer might influence written English and suggested that negative language transfer might occur when the realization of specific language features differed between the first language and written English. For instance, one feature is the realization of the copula "be". In ASL the copula "be" is often not lexicalized. Thus, negative language transfer might predict omission errors resulting from not lexicalizing the copula "be" in the written English of ASL signers. While we concentrate here on errors from the ASL population, the errors identified are likely to be found in learners coming from first languages other than ASL as well. This would be the case if the first language has features in common with ASL. For instance the missing copula "be" is also a common error in the writing of native Chinese speakers since Chinese and ASL share the feature that the copula "be" is often not lexicalized. Thus, the examples seen here will generalize to other languages.

In the following we describe some classes of errors which we uncovered (and attempt to "explain" why an ASL native might come to make these errors).

\subsection{Constituent Omissions}

Learners of English as a second language (ESL) omit constituents for a variety of reasons. One error that is common for many ASL learners is the dropping of determiners. Perhaps because ASL does not have a determiner system similar to that of English, it is not unusual for a determiner to be omitted as in:

(1) I am - transfer student from ....

These errors can be flagged reasonably well when they are syntactic (and not pragmatic) in 
nature and do not pose much additional burden on the parser/grammar.

However, missing main verbs (most commonly missing copulas) are also common in our writing samples:

(2) Once the situation changes they _ different people.

One explanation for this (as well as other missing elements such as missing prepositions) is that copulas are not overtly lexicalized in ASL because the copula (preposition) is gotten across in different ways in ASL. Because the copula (preposition) is realized in a radically different fashion in ASL, there can be no positive language transfer for these constructions.

In addition to omitting verbs, some NPs may also be omitted. It has been argued (see, for example (Lillo-Martin, 1991)) that ASL allows topic NP deletion (Huang, 1984) which means that topic noun phrases that are prominent in the discourse context may be left out of a sentence. Carrying this strategy over to English might explain why some NPs are omitted from sentences such as:

(3) While living at college I spend lot of money because - go out to eat almost everyday.

Mal-rules written to handle these errors must capture missing verbs, NPs, and prepositions. The grammar is further complicated because ASL natives also have many errors in relative clause formation including missing relative pronouns. The possibility of all of these omissions causes the parser to explore a great number of parses (many of which will complete successfully).

\subsection{Handling Omissions}

As we just saw, omissions are frequent in the writing of ASL natives and they are difficult to detect using the mal-rule formalism. To clearly see the problem, consider the following two sentences, which would not be unusual in the writing of an ASL native.

(4) The boy happy.

(5) Is happy.

As the reader can see, in (4) the main verb "be" is omitted, while the subject is missing in (5).

To handle these types of sentences, we included in our grammar mal-rules like the following:

(6) VP(error + ) $\rightarrow$ AdjP

(7) $\mathrm{S}($ error +$) \rightarrow$ VP
A significant problem that arises from these rules is that a simple adjective is parsed as an $\mathrm{S}$ even if it is in a normal, grammatical sentence. This behavior leads to many extra parses, since the $\mathrm{S}$ will be able to participate in lots of other parses. The problem becomes much more serious when the other possible omissions are added into the grammar. However, closer examination of our writing samples indicates that, except for determiners, our users generally leave out at most one word (constituent) per sentence. Thus it is unlikely that "happy" will ever be an entire sentence. We would like this fact to be reflected in the analyses explored by the parser. However, a traditional bottom-up context-free parser has no way to deal with this case, as there is no way to block rules from firing as long as the features are capable of unification.

One possibility would be to allow the (error + ) feature to percolate up through the parse. Any rule which introduces the (error + ) feature could then be prevented from having any children specified with (error +). However, this solution would be far too restrictive, as it would restrict the number of errors in a sentence to one, and many of the sentences in our ASL corpus involve multiple errors.

Recall, however, that in our analysis we found that (except for determiners) our writing samples did not contain multiple omission errors in a sentence. Thus another possibility might be to percolate an error feature associated with omissions only-perhaps called (missing + ).

Upon closer inspection, this solution also has difficulties. The first difficulty has to do with implementing the feature percolation. For instance, for a VP to be specified as (missing +) whenever any of its sub-constituents has that feature, one would need to have separate rules raising the feature up from each of the subconstituents, as in the following:

(8) VP(missing ?a) $\rightarrow$ V NP NP(missing ?a)

(9) VP(missing ?a) $\rightarrow$ V NP(missing ?a) NP

(10) VP(missing ?a) $\rightarrow$ V(missing ?a) NP NP

This would cause an unwarranted increase in the size of the grammar, and would also cause an immense increase in the number of parses, since three VPs would be added to the chart, one for each of the rules.

At first glance it appears that this problem can be overcome with the use of "foot features," which are included in the parser we are using. A foot feature moves features from any child to the parent. For example, for a foot feature $F$, if one child has a specification for $F$, it will be passed 
on to the parent. If more than one child is specified for $F$, then the values of $F$ must unify, and the unified value will be passed up the parent. While the use of foot features appears to make the feature percolation easier, it will not allow the feature to be used as desired. In particular, we need to have the feature percolated only when it has a positive value and only when that value is associated with exactly one constituent on the right-hand side of a rule. The foot feature as defined by the parser would allow the percolation of the feature even if it were specified in more than one constituent.

A further complication with using this type of feature propagation arises because there are some situations where multiple omission errors do occur, especially when determiners are omitted. ${ }^{1}$ Consider the following example taken from our corpus where both the main verb "be" and a determiner "the" are omitted.

(11) Student always bothering me while I am at dorm.

(Corrected) Students are always bothering me while I am at the dorm.

Our solution to the problem involves using procedural attachment. The parser we are using builds constituents and stores them in a chart. Before storing them in the chart, the parser can run arbitrary procedures on new constituents. These procedures, specified in the grammar, will be run on all constituents that meet a certain pattern specified by the grammar writer.

Our procedure amounts to specifying an alternative method for propagating the (missing +) feature, which will still be a foot feature. It will be run on any constituent that specifies (missing + ). The procedure can either delete a constituent that has more than one child with (missing +), or it can alter the (missing +) feature on the constituent in the face of determiner omissions (as discussed in footnote 1). By using a special procedure to implement the feature percolation, we will be able to be more flexible in where we allow the "missing" feature to percolate.

\subsection{Syntactic Feature Addition}

For this system to properly model language acquisition, it must also model the addition (and possible subtraction) of syntactic features in the lexicon and grammar of the learner. For instance, ASL natives have a great deal of difficulty with many of the agreement features in

\footnotetext{
${ }^{1}$ While our analysis so far has only indicated that determiner omissions have this property, we do not want to rule out the possibility that other combinations of omission errors might be found to occur as well.
}

English. As a concrete example, this population frequently has trouble with the difference between "other" and "another". They frequently use "other" in a singular NP, where "another" would normally be called for. We hypothesize that this is partly a result of their not understanding that there is agreement between NPs and their specifiers (determiners, quantifiers, etc.). Even if this is recognized, the learners may not have the lexical representations necessary to support the agreement for these two words. $^{2}$ Thus, the most accurate model of the language of these early learners involves a lexicon with impoverished entries - i.e. no person or number features for determiners and quantifiers. Such an impoverished lexicon would mean that the entries for the two words might be identical, which appears to be the case for these learners.

There are at least two reasons for not using this sort of impoverished lexicon. Firstly, it would require having multiple lexicons (some impoverished, others not), with the system needing to determine which to use for a given user. Secondly, it would not allow grammatical uses of the impoverished items to be differentiated from ungrammatical uses. With an impoverished lexicon, any use (grammatical or not) of "other" or "another" would be flagged as an error, since it would involve using a lexical entry that does not have all of the features that the standard entry has. Since the lexical item would not have the agr specification, it could not match the rule that requires agreement between determiners and nouns.

\subsubsection{Implementation}

For these reasons, we decided not to use different lexical entries to model the different stages of acquisition. Instead, we use mal-rules, the same mechanism that we are using to model syntactic changes. A standard (grammatical) DP (Determiner Phrase) rule has the following format:

(12) DP (agr ?a) $\rightarrow$ Det (agr ?a) NP (agr ?a)

We initially tried simply eliminating the references to agreement between the NP and the determiner, as in the following mal-rule:

(13) DP (error +) (agr ?a) $\rightarrow$ Det NP (agr ?a)

This has the advantage of flagging any deviant DPs as having the error feature, since ungrammatical DPs will trigger the mal-rule (13), but won't trigger (12). However, a grammatical

\footnotetext{
2 "Another" and "other" are not separate lexical items in ASL.
} 
DP (e.g. "another child") fires both the malrule (13) and the grammatical rule (12). Not only did this behavior cause the parser to slow down very significantly, since it effectively doubled the number of DPs in a sentence, but it also has the potential to report an error when one does not exist. We also briefly considered using impoverishment rules on specific categories. For example, we could have used a rule stating that determiners have all possible agreement values. This has the effect of eliminating agreement as a barrier to unification, much as would be expected if the learner has no knowledge of agreement on determiners. However, this solution has a problem very similar to that of the previous possible solution: all determiners in the input could suddenly have two entries in the chart - one with the actual agreement, one with the impoverished agreement. These would then both be used in parsing, leading to another explosion in the number of parses.

We finally ended up building a set of rules that matches just the ungrammatical possibilities, i.e. they do not allow a grammatical structure to fire both the mal-rule and the normal rule. The present set of rules for determinerNP agreement include the following: ?a)

(14) DP (agr ?a) $\rightarrow$ Det (agr ?a) NP (agr

(15) DP (agr s) (error + ) $\rightarrow \operatorname{Det~(agr~(?!~a~}$ s)) NP (agr s)

(16) DP (agr p) (error + ) $\rightarrow \operatorname{Det}($ agr (?! a p)) NP(agr p)

This solution required using the negation operator "?" present in our parser to specify that a Det not allow singular/plural agreement. However, this feature is limited in the present implementation to constant values, i.e. we can't negate a variable. This solution achieves the major goal of not introducing extraneous parses for grammatical constituents. However, it achieves this goal at some cost. Namely, we are forced to increase the number of rules in order to accomplish the task.

\subsubsection{Future plans}

We are presently working on the implementation of a variant of unification that will allow us to do the job with fewer rules. The new operation will work in the following sort of rule:

(17) DP (agr ?a) $\rightarrow$ Det (agr ?! a) NP (agr ?a)

This rule will be interpreted as follows: the agr values between the DP and the NP will be the same, and none of the values in Det will be allowed to be in the agreement values for the NP and the DP. This will allow the rule to fire precisely when there are no possible ways to unify the values between the Det and the NP, i.e. none of the agr values for the Det will be allowed in the variable ?a. Thus, this rule will only fire for ungrammatical constructions.

\section{Grammar Coverage/User Interface}

The ICICLE grammar is a broad-coverage grammar designed to parse a wide variety of both grammatical sentences and sentences containing errors. It is built around the COMLEX Syntax 2.2 lexicon (Grishman et al., 1994), which contains approximately 38,000 different syntactic head words. We have a simple set of rules that allows for inflection, thereby doubling the number of noun forms, while giving us three to four times as many verb forms as there are heads. Thus we can handle approximately 40,000 noun forms, 8,000 adjectives, and well over 15,000 verb forms. In addition, unknown words coming into the system are assumed to be proper nouns, thus expanding the number of words handled even further.

The grammar itself contains approximately 25 different adjectival subcategorizations, including subcategorizations requiring an extraposed structure (the "it" in "it is true that he is here"). We also include half a dozen noun complementation types. We have approximately 110 different verb complementation frames, many of which are indexed for several different subcategorizations. The grammar is also able to account for verb-particle constructions when the verb is adjacent to the particle, as well as when they are separated (e.g. "I called him up").

Additionally, the grammar allows for various different types of subjects, including infinitivals with and without subjects ("to fail a class is unfortunate", "for him to fail the class is irresponsible"). It handles yes/no questions, whquestions, and both subject and object relative clauses.

The grammar has only limited abilities concerning coordination - it only allows limited constituent coordination, and does not allow non-constituent coordination (e.g. "I saw and he hit the ball") at all. It is also fairly weak in its handling of adjunct subordinate clauses. The population we are concerned with also has significant trouble with this, in particular there is a strong propensity towards over-using "because". Adverbs are also problematic, in that the system is not yet able to differentiate what position a given adverb should be able to take in a sentence, thus no errors in adverb placement 
can be flagged. We are presently in the process of integrating a new version of the lexicon that includes features specifying what each adverb can attach to. Once this is done, we expect to be able to process adverbs quite effectively.

The user interface presently consists of a main window where the user can input the text and control parsing, file access, etc. After parsing, the sentences are highlighted with different colors corresponding to different types of errors. When the user double-clicks on a sentence, a separate "fix-it" window is displayed with the sentence in question, along with descriptions of the errors. The user can click on the errors and the system will highlight the part of the sentence where the error occurred. For example, in the sentence "I see a boys", only "a boys" will be highlighted. The "fix-it" window also allows the user to change the sentence and then re-parse it. If the changes are acceptable to the user, the new sentence can be substituted back into the main text.

\section{Evaluation of Error Recognition}

An evaluation of the grammar was conducted on a variety of sentences pulled from the corpus of ASL natives. The corpus contains essays written by ASL natives which is annotated with references to different types of errors in the sentences. The focus for this paper was on recognition of agreement-type problems, and as such we pulled out all of the sentences that had been marked with the following errors:

- NUM: Number problems, which are typically errors in subject-verb agreement

- ED: extra determiner

- MD: missing determiner for an NP that requires a determiner

- ID: incorrect determiner

In addition to testing sentences with these problems, we also tested fully grammatical sentences from the same corpus, to see if we could correctly differentiate between grammatical and ungrammatical sentences that might be produced by our target user group.

After gathering the sentences from the database, we cut them down to mono-clausal sentences wherever possible, due to the fact that the handling of adjunct clauses is not yet complete (see §4). An example of the type of sentence that had to be divided is the following:

(18) They should communicate each other because the communication is very important to understand each other.
This sentence was divided into "They should communicate each other" and "the communication is very important to understand each other." In addition to separating the clauses, we also fixed the spelling errors in the sentences to be tested since spelling correction is beyond the scope of the current implementation.

\subsection{Results for Ungrammatical Sentences}

We ended up with 79 sentences to test for the determiner and agreement errors. Of these 79 sentences, $44(56 \%)$ parse with the expected type of error. Another 23 (29\%) have no parses that cover the entire sentence, and $12(15 \%)$ parse as having no errors at all.

A number of the sentences that had been flagged with errors in the database were actually grammatical sentences, but were deemed inappropriate in context. Thus, sentences like the following were tagged with errors in the corpus:

(19) I started to attend the class last Saturday.

It was evident from the context that this sentence should have had "classes" rather than "the class." Of the 12 sentences that were parsed as error-free, five were actually syntactically and semantically acceptable, but were inappropriate for their contexts, as in the previous example. Another four had pragmatic/semantic problems, but were syntactically well-formed, as in

\section{(20) I want to succeed in jobs anywhere.}

Thus, there are really only three sentences that do not have a parse with the appropriate error. Since this parser is a syntactic parser, it should not be expected to find the semantic/pragmatic errors, nor should it know if the sentence was inappropriate for its context in the essay. If we eliminate the nine sentences that are actually grammatical in isolation, we are left with 70 sentences, of which $44(63 \%)$ have parses with the expected error, three (4\%) are wrongly accepted as grammatical, and $23(33 \%)$ do not parse.

In terms of evaluating these results for the purposes of the system, we must consider the implications of the various categories. $63 \%$ would trigger tutoring, and $33 \%$ would be tagged as problematic, but would have no information about the type of error. In only $4 \%$ of sentences containing errors would the system incorrectly indicate that no errors are present.

5.2 Results for Grammatical Sentences We also tested the system on 101 grammatical sentences that were pulled from the same corpus. These sentences were modified in the same 
way as the ungrammatical ones, with multiclausal sentences being divided up into monoclausal sentences. Of these 101 sentences, 89 $(88 \%)$ parsed as having no errors, $3(3 \%)$ parsed with errors, and the remaining $8(8 \%)$ did not parse.

The present implementation of the grammar suffers from poor recognition of coordination, even within single clauses. Five of the eleven sentences that did not return an error-free parse suffered from this limitation. We expect to be able to improve the numbers significantly by including in the grammar some recognition of punctuation, which, due to technical problems, is presently filtered out of the input before the parser has a chance to use it.

\section{Conclusions and Future Work}

Future work will include extending the grammar to better deal with coordination and adjunct clauses. We will also continue to work on the negation operator and the propagation of the missing feature discussed above. In order to cut down on the number of parses, as well as to make it easier to decide which is the appropriate parse to correct, we have recently switched to a best-first parsing strategy. This should allow us to model which rules are most likely to be used by a given user, with the mal-rules corresponding to the constructions currently being acquired having a higher probability than those that the learner has already mastered. However, at the moment we have simply lowered the probabilities of all mal-rules, so that any grammatical parses are generated first, followed by the "ungrammatical" parses.

As we have shown, this system does a good job of flagging ungrammatical sentences produced by the target population, with a high proportion of the flagged sentences containing significant information about the type and location of the error. Our continuing work will hopefully improve these percentages, and couple this recognition component with an intelligent tutoring phase.

\section{References}

James Allen. 1995. Natural Language Understanding, Second Edition. Benjamin/Cummings, CA.

N. Bailey, C. Madden, and S. D. Krashen. 1974. Is there a 'natural sequence' in adult second language learning? Language Learning, 24(2):235-243.

Vivian Cook. 1993. Linguistics and Second Language Acquisition. Macmillan Press Ltd, London.
Heidi C. Dulay and Marina K. Burt. 1974. Natural sequences in child second language acquisition. Language Learning, 24:37-53.

Rod Ellis. 1994. The Study of Second Language Acquisition. Oxford University Press, Oxford.

Ralph Grishman, Catherine Macleod, and Adam Meyers. 1994. Comlex syntax: Building a computational lexicon. In Proceedings of the 15th International Conference on Computational Linguistics, Kyoto, Japan, July. Coling94.

C.-T. James Huang. 1984. On the distribution and reference of empty pronouns. Linguistic Inquiry, 15(4):531-574, Fall.

David Ingram. 1989. First Language Acquisition: Method, Description, and Explanation. Cambridge University Press, Cambridge; New York.

Stephen Krashen. 1981. Second Language Acquisition and Second Language Learning. Pergamon Press, Oxford.

Diane C. Lillo-Martin. 1991. Universal Grammar and American Sign Language. Kluwer Academic Publishers, Boston.

Lisa N. Michaud and Kathleen F. McCoy. 1998. Planning tutorial text in a system for teaching english as a second language to deaf learners. In Proceedings of the 1998 AAAI Workshop on Integrating Artificial Intelligence and Assistive Technology, Madison, Wisconsin, July.

Lisa N. Michaud. 1998. Tutorial response generation in a writing tool for deaf learners of english. In Proceedings of the Fifteenth National Conference on Artificial Intelligence (poster abstract), Madison, Wisconsin, July.

L. Selinker. 1972. Interlanguage. International Review of Applied Linguistics, 10:209-231.

D. Sleeman. 1982. Inferring (mal) rules from pupil's protocols. In Proceedings of ECAI-82, pages 160-164, Orsay, France. ECAI-82.

Linda Z. Suri and Kathleen F. McCoy. 1993. A methodology for developing an error taxonomy for a computer assisted language learning tool for second language learners. Technical report TR-93-16. Dept. of CIS, University of Delaware.

Lev Semenovich Vygotsky. 1986. Thought and Language. MIT Press, Cambridge, MA.

Ralph M. Weischedel, Wilfried M. Voge, and Mark James. 1978. An artificial intelligence approach to language instruction. Artificial Intelligence, 10:225-240. 